\title{
Tratamientos de protección superficial de materiales de construcción por nuevos materiales híbridos organo-inorgánicos multifuncionales
}

\author{
J. L .OTEO', M. R. ELVIRA ${ }^{1,2}$, L. ALONSO', J. RUBIO' \\ 1. Departamento de Química-Física de Superficies y Procesos. Instituto de Cerámica y Vidrio, CSIC; \\ C/ Kelsen s/n Campus de Cantoblanco. UAM.; C.P. 28049, Madrid. \\ 2. Departamento de Química Analítica e Ingeniería Química. Facultad de Química. \\ Universidad de Alcalá de Henares. Ctra. N II, km 33,600. 28870, Madrid.
}

\begin{abstract}
En este trabajo se presentan los resultados de las investigaciones llevadas a cabo para evaluar el comportamiento de un material híbrido orgánico-inorgánico (HOI) como recubrimiento protector de distintos materiales de construcción. La caracterización mineralógica, estructural y textural de los materiales de construcción estudiados (granito, mármol y arenisca) se llevó a cabo mediante las técnicas: difracción de rayos X (DRX), microscopía electrónica de barrido (MEB), reflectancia total atenuada (ATR) y porosimetría de mercurio. Por otro lado, la caracterización del recubrimiento antivandálico se llevó a cabo mediante ATR y MEB, evaluando su capacidad protectora contra pinturas realizadas por aerógrafo o rotulador, según la mayor o menor facilidad con que se elimina la pintura por limpieza con gamuza.

La estabilidad del recubrimiento en el material inorgánico se estudió mediante ensayos acelerados de resistencia a la lluvia y, en el caso del mármol, a la radiación UV. La variación del color experimentada por la superficie después de ser recubierta se estudió, en todos los casos, por colorimetría.
\end{abstract}

Palabras clave: antivandálico, hibrido, recubrimiento, envejecimiento.

\section{Surface protection treatments of building materials by new organic-inorganic hybrid multifuncional materials}

The behavior of an organic-inorganic hybrid material (HOI) as protective coating of different building materials has been evaluated in this work.

The mineralogical, structural and textural characterization of the inorganic building materials (granite, marble and sandstone) was carried out by: x-ray diffraction (XRD), scanning electron microscopy (SEM), attenuated total reflectance (ATR) and mercury porosimetry. On the other hand, the protective coating was characterized by ATR and SEM, evaluating its protective capacity against paintings made by aerograph or labeller, according to the greater or smaller facility whereupon eliminates the painting by means of cleaning with a cloth.

The stability of the coating on the inorganic materials was studied by accelerated tests of rain and UV radiation. Color variation after coating was studied, in all the cases, by colorimetry.

Keywords: antigraffiti, hybrid, coating, ageing.

\section{INTRODUCCIÓN}

Las fachadas de edificios están expuestas a gran cantidad de agentes de deterioro tanto de origen natural (heladas, agentes biológicos, eflorescencias y cristalización de sales, solubilización de los componentes del material constituyente, etc., (1-7) como de origen antropogénico (agentes contaminantes de la atmósfera, vandalismo, etc). Para evitar este deterioro, se han desarrollado un gran número de compuestos cuya finalidad es recubrir la fachada y protegerla de dichos agentes nocivos (3, 8-12).

El desarrollo de nuevos materiales híbridos orgánicoinorgánicos (13), con determinadas características $(14,8)$, hacen posible su empleo como recubrimiento de fachadas. Entre las más importantes, en cuanto a su aplicación como recubrimiento, se encuentran la transparencia, con lo que no se altera el aspecto y color del material de construcción original, y la estabilidad e inalterabilidad frente a la acción de agentes atmosféricos, esto es, que no sean fotodegradables, ni lixiviables y/o hidrolizables bajo la acción del agua de lluvia $(9,10,15)$. Por esto, resulta siempre necesario evaluar las características y propiedades de los recubrimientos protectores obtenidos, mediante ensayos de envejecimiento acelerado realizados por simulación de condiciones extremas de luz UV y lluvia.

El objetivo de este trabajo es conseguir a partir de un material híbrido orgánico-inorgánico (HOI) ternario de dos formadores de red y una cadena polimérica, un recubrimiento que proporcione al material de construcción propiedades protectoras antivandálicas, es decir, que sea inalterable frente a las manchas de pintura, sin que haya modificación de las características del soporte original, y que presente un buen comportamiento frente a las alteraciones climáticas. 


\section{PROCEDIMIENTO EXPERIMENTAL}

\subsection{Síntesis}

La síntesis del material híbrido orgánico-inorgánico (HOI) se realizó mediante el método desarrollado por Oteo, Rubio y Rubio (16), a partir de derivados de silicio (metoxisilanos), modificados convenientemente para conseguir laincorporación de un segundo formador de red inorgánica como pueden ser $\mathrm{Ti}, \mathrm{Zr}, \mathrm{B}, \mathrm{Al}$, etc., utilizando un propóxido u otro compuesto orgánico de estos elementos como precursor. La relación entre reactivos empleada en el HOI específico utilizado en este trabajo ha sido: $60 / 40$.

Componentes inorgánicos/ componentes orgánicos:

Porcentaje de componente inorgánico de red (no silíceo): $10 \%$.

Relación molar disolvente / componente silíceo: 5 .

Cuando los reactivos son incorporados al matraz, provisto de refrigerante de reflujo, agitación mecánica y calentado en un baño entre $35-65{ }^{\circ} \mathrm{C}$, dan lugar a un sol que, terminado el tiempo de reacción, se almacena hasta su aplicación en un recipiente herméticamente cerrado, para evitar su hidrólisis y posterior gelificación por acción de la humedad ambiental y evaporación del disolvente. El tiempo de reacción utilizado varía entre 12-72 horas, según la parametrización del proceso y su cinética, estudiada por Elvira y col. (17)

\subsection{Materiales}

Los materiales soporte empleados en este trabajo han sido mármol, granito y arenisca que, dada su distinta composición y características, presentan comportamientos muy diferentes ante el mismo recubrimiento protector. Dichos materiales fueron suministrados por CPA, S.A., (Conservación del Patrimonio Arquitectónico, S.A. Burgos). La composición mineralógica, estructura y textura de estos materiales se estudió por DRX, MEB y ATR. Los difractogramas DRX se obtuvieron por la técnica de polvo mediante un difractómetro Siemens modelo D5000 con un generador Kristalloflex 710 operando a un voltaje de $40 \mathrm{kV}$ y una intensidad de $20 \mathrm{~mA}$. El barrido aplicado a los difractogramas fue de 10 a $70^{\circ}(2 \theta)$. Por otro lado la microestructura se observó mediante un microscopio Zeiss DSM950 trabajando a $20 \mathrm{kV}$. Los espectros ATR fueron obtenidos en un equipo FT-IR 1760X Perkin-Elmer al cual se le acopló un dispositivo de reflectancia con punta de diamante también Perkin-Elmer. Por otro lado, la porosidad se estudió mediante porosimetría de mercurio utilizando un equipo Micromeritics Autopore II-9215 en el intervalo de presiones de intrusión desde 0.2 a $202 \mathrm{MPa}$, valores que corresponden a tamaños de poro de $0.3 \mathrm{~mm}$ a $6.2 \mathrm{~nm}$, respectivamente. El modelo de intrusión utilizado fue el de Washburn suponiendo que los poros poseen forma cilíndrica.

La caracterización del tratamiento protector y su capacidad como recubrimiento en el soporte se llevó a cabo por ATR. El grado de penetración del HOI en la superficie inorgánica y su homogeneidad en la dispersión o distribución superficial se examinó mediante MEB.

TABLA I. POROSIDAD Y TAMAÑO MEDIO DE PORO DE LOS MATERIALES DE PARTIDA.

\begin{tabular}{|c|c|c|}
\hline Material & Porosidad $(\%)$ & Tamaño de Poro $(\boldsymbol{\mu} \mathbf{m})$ \\
\hline Arenisca & 36.89 & 24.98 \\
\hline Granito & 3.9 & 14.19 \\
\hline Mármol & $<1$ & 0.16 \\
\hline
\end{tabular}

\subsection{Recubrimiento y comportamiento antivandálico}

La aplicación del recubrimiento con el material HOI de las superficies pétreas se realizó mediante pulverización con pistola eléctrica, a una distancia de $20 \mathrm{~cm}$ y durante $5 \mathrm{~s}$. A continuación, los materiales tratados se secaron a temperatura ambiente hasta la gelificación del recubrimiento. Pasadas los 2 días el recubrimiento alcanza peso constante con lo que se puede decir que ya está consolidado.

El estudio de la funcionalidad antivandálica se llevó a cabo de la siguiente manera: sobre las piedras impregnadas y secas, se pulverizó a una distancia de $10 \mathrm{~cm}$, durante $3 \mathrm{~s}$ simulando una pintada con esmalte acrílico Novasol Spray. Después la pintada se secó a temperatura ambiente y, posteriormente, se limpió con un paño de celulosa. La variación de los parámetros cromáticos de los distintos soportes pétreos, antes y después de la aplicación del recubrimiento, se analizó con un espectrofotómetro Minolta-2600d con iluminante D65 (equivalente a luz diurna), ángulo de observador de $10^{\circ} \mathrm{y}$ máscara de medida de $3 \mathrm{~cm}$ de diámetro. Los valores de los parámetros cromáticos corresponden al promedio de los resultados obtenidos en 5 puntos diferentes de la superficie.

Para discernir los cambios cromáticos producidos al recubrir la superficie se puede emplear la elipse de tolerancias la cual se construye a partir de las coordenadas cromáticas empleando como espacio de color el sistema CIELab. Este sistema corresponde a una esfera de color cuyas coordenadas $\mathrm{L}^{*}, \mathrm{a}^{*} \mathrm{y} \mathrm{b}^{*}$ determinan un punto en dicho espacio. En el eje $\mathrm{a}^{*}$, de escala $+60-60$, se representa el color verde-rojo, en el eje $b^{*}$, también de escala $+60-60$, se representa el color azul-amarillo y en el eje L, de escala 0 a 100, representa la luminosidad, negro-blanco. El centro de la elipse se corresponde con los valores $\mathrm{a}^{*} \mathrm{y} \mathrm{b}^{*}$ de la muestra patrón y los ejes de dicha elipse se calculan a partir de las siguientes ecuaciones (18):

\section{$S_{t}=\frac{0.040975^{*} L}{\left(1+0.01765^{*} L^{L}\right)}$ sit $L^{*}>16$ o}

\section{$s,=0511$ as $L \leq 16$}

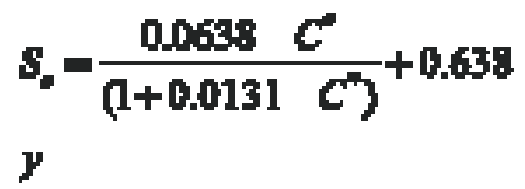

$$
s_{1}=(f T+1-f) S_{c} \text { ands }
$$

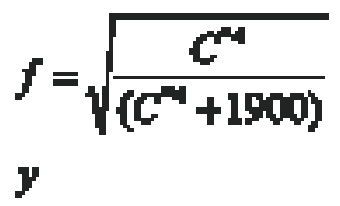

$$
\begin{aligned}
& T=0.56+\left|0.2000\left(h^{*}+160\right)\right| \operatorname{sil} 164 \mathrm{pph} h^{*} 345^{\prime} \\
& T=0.36+\left|0.4000\left(h^{*}+35\right)\right|
\end{aligned}
$$


A continuación se determina el cambio de color del soporte debido al recubrimiento obteniéndose los valores de $\mathrm{L}_{\mathrm{m}^{\prime}}^{*} \mathrm{a}_{\mathrm{m}}^{*} \mathrm{y}$ $\mathrm{b}_{\mathrm{m}}^{*}$. Si estos nuevos valores de color se encuentran dentro de la elipse de tolerancias puede decirse que el cambio de color superficial no es distinguible por el ojo humano, mientras que si dichos valores caen fuera de la misma, el cambio de color sería el suficiente como para que el ojo humano lo detectase.

\subsection{Ensayos de envejecimiento}

\subsubsection{Ensayo de resistencia a la lluvia}

En este ensayo, cuyo objetivo es determinar la resistencia del recubrimiento al arrastre y/o hidrólisis por efecto de la lluvia, se han empleado probetas de $5 \times 5 \times 1 \mathrm{~cm}^{3}$ de granito, mármol y arenisca, impregnadas de la manera descrita con el material híbrido. Éstas probetas se sometieron a 2 y 10 ciclos de lluvia de $30 \mathrm{~min} /$ ciclo, con un volumen de 8,23 1 en cada ciclo. Los 2 ciclos, corresponden a un clima templado continental, con precipitaciones de $224 \mathrm{l} / \mathrm{m}^{2}$ anuales, mientras que los 10 ciclos corresponden a un clima muy húmedo, de $1120 \mathrm{l} / \mathrm{m}^{2}$ de precipitaciones anuales.

Los ciclos de lluvia se han simulado en la cámara de corrosión Kesternich (Serie VCK), provista de un dispositivo que permite la salida del agua, simulando lluvia, que cae sobre los materiales a ensayar. Después de cada ciclo, se secan las muestras a temperatura ambiente y se evalúa tanto su comportamiento antivandálico (por el método descrito anteriormente), como la posible variación cromática observada en las superficies de los materiales inorgánicos soporte.

\subsubsection{Envejecimiento por radiación UVI}

En el estudio de la variación de color del recubrimiento por interacción con radiación UV (envejecimiento) se empleó únicamente el mármol siendo descartados el granito y la arenisca debido su heterogeneidad (el granito presenta diferentes colores originados por los distintos minerales que lo componen, y la arenisca presenta poros que dan lugar a inhomogeneidades superficiales). La dimensión de las probetas de mármol fue de 2,3×5x0,9 cm³. El ensayo se llevó a cabo en una cámara climática sometiendo sendas probetas a ciclos de luz UV de 5, 10, 20 y 200 horas en una estufa AFA tipo SUNLAMP, termostatizada a $35^{\circ} \mathrm{C}$. Después de transcurrido el tiempo de cada ciclo se realizaron las correspondientes medidas cromáticas.

\section{RESULTADOS Y DISCUSIÓN}

\subsection{Caracterización de los materiales de partida}

En la figura 1 se presentan los difractogramas DRX junto con el análisis mineralógico cualitativo correspondiente. El granito está compuesto de cuarzo y feldespato cálcico (plagioclasa); el mármol es calcita y, por último, la arenisca está compuesta por calcita, cuarzo y dolomita.

Los resultados obtenidos por porosimetría de mercurio se muestran en la Figura 2 en donde puede comprobarse como la arenisca presenta una amplia distribución de poros (desde 0.05 a 100 micrómetros), a continuación está el granito que presenta una distribución menos amplia (entre 0.2 y 50 micrómetros) y finalmente el mármol presenta una muy pequeña distribución de poros. Los valores medios de tamaño de poro y porosidad

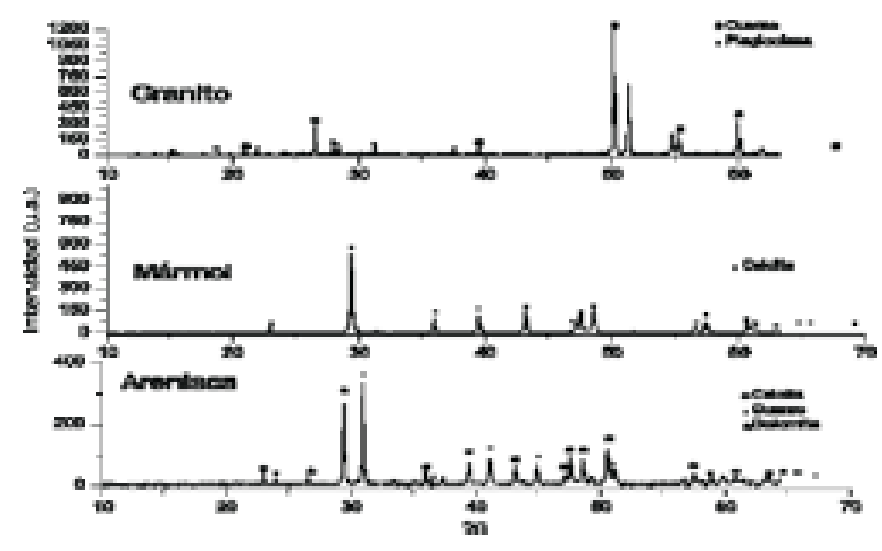

Fig. 1.- Espectros de difracción de rayos $\mathrm{X}$ de los materiales pétreos originales.

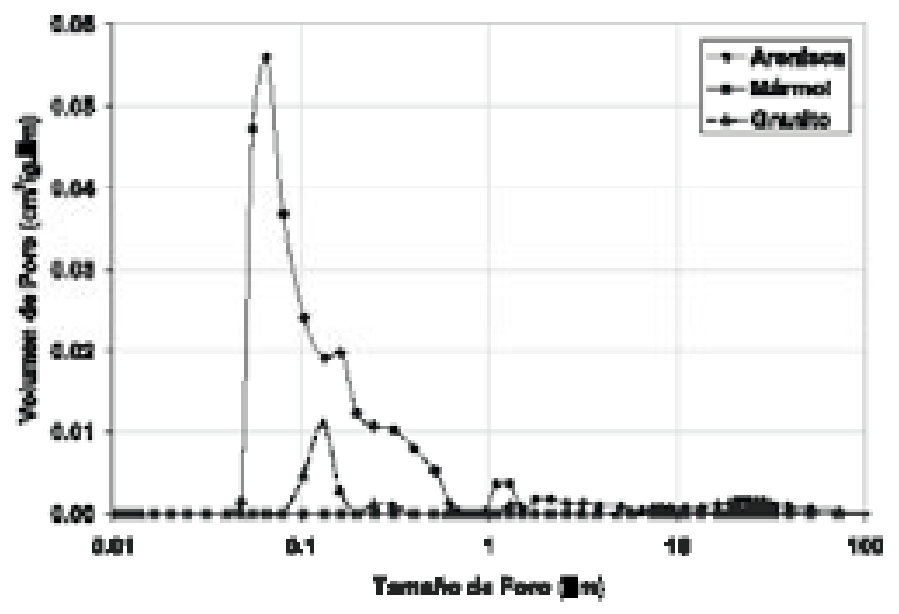

Fig. 2.- Porosidad de los materiales pétreos originales.

se recogen en la Tabla I en la cual se puede comprobar como la arenisca posee una porosidad próxima al $37 \%$, el granito del $4 \%$, mientras que la del mármol es inferior al $1 \%$. Como era de esperar, el tamaño de poro (determinado a partir de la curva de distribución de volúmenes de poro) aumenta en el sentido mármol $<$ granito $<$ arenisca, es decir de acuerdo con la porosidad y con la distribución de poros de cada material.

\subsection{Caracterización de los materiales impregnados}

Los espectros ATR para los distintos materiales empleados se muestran en la figura 3 , junto con los correspondientes a los materiales recubiertos y con el del HOI de partida. Comparando los espectros del material inorgánico soporte original y del recubierto se puede observar cómo, en el caso del granito, sólo se observan las bandas pertenecientes al material híbrido, como son las correspondientes a los enlaces $\mathrm{Si}-\mathrm{CH}_{3^{\prime}}\left(1257 \mathrm{~cm}^{-1}\right)$ y Si-O-Si $\left(1008 \mathrm{~cm}^{-1}\right)$. Este hecho sugiere un recubrimiento superficial homogéneo y uniforme por parte del HOI.

En el caso del mármol, las bandas correspondientes a la calcita no son apreciables una vez recubierta su superficie, sólo se distinguen las bandas del HOI anteriormente señaladas, repitiéndose la situación anterior. Por último, en el espectro de la arenisca se observan las bandas características del HOI muy débiles, lo que indica que el HOI no se encuentra en la superficie sino que ha penetrado en el material, debido a su 


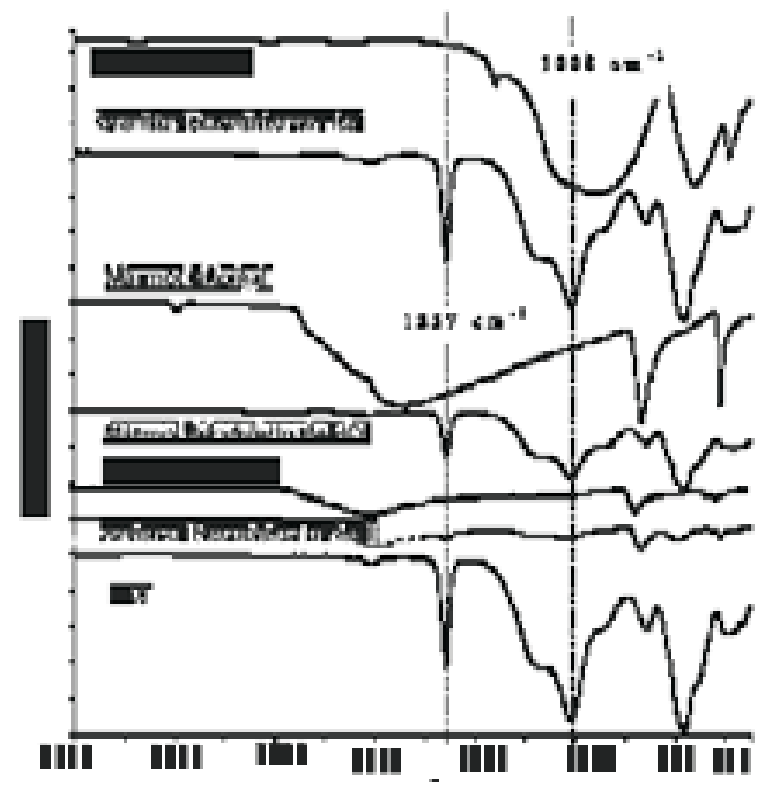

Fig. 3.- Espectros ATR del ormosil, materiales pétreos originales y recubiertos con HOI.

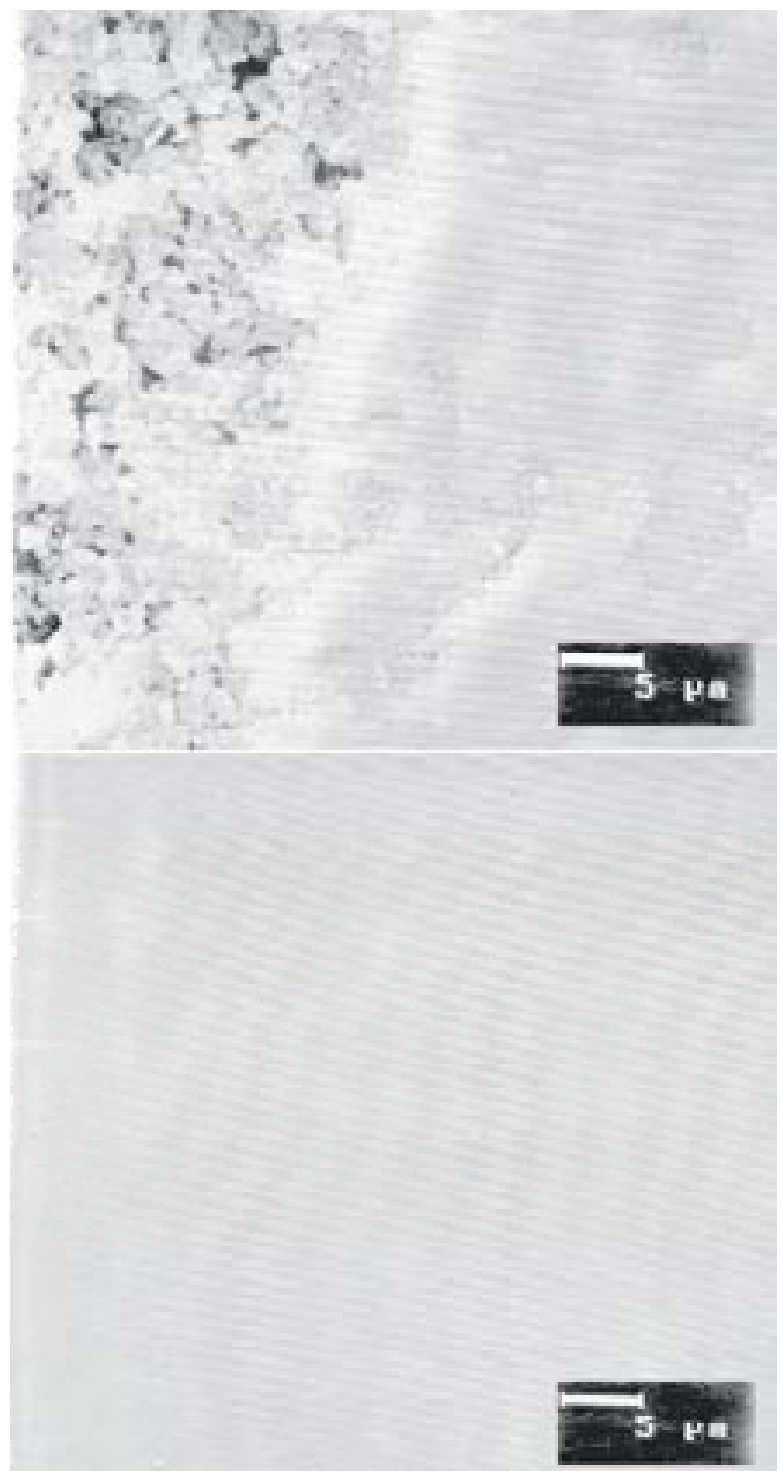

Fig. 4.- Fotografías de microscopía electrónica de barrido de granito. (a) original; (b) recubierto con HOI.
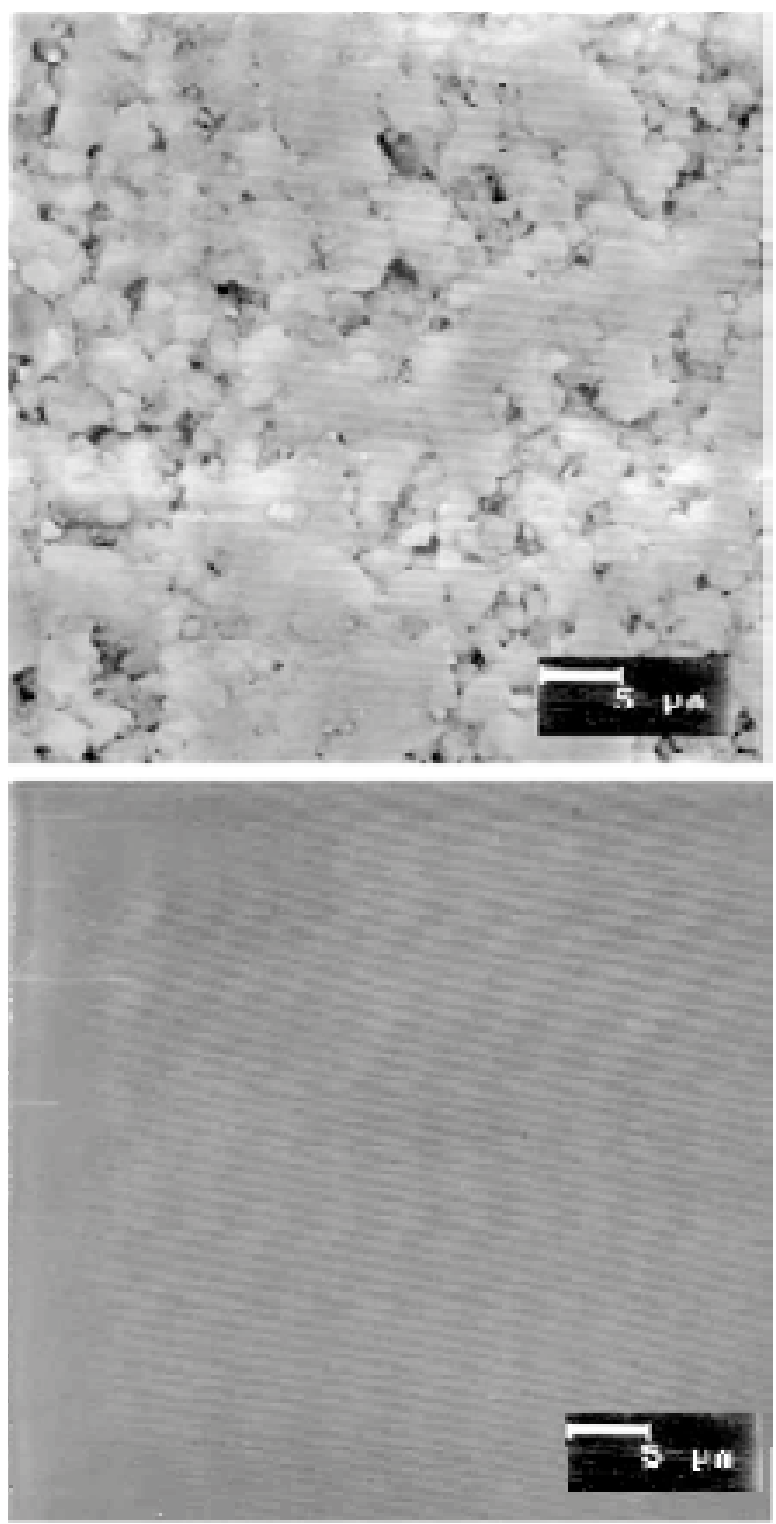

Fig. 5.- Fotografías de microscopía electrónica de barrido de mármol. (a) original; (b) recubierto con HOI.

mayor porosidad, con lo que su capacidad protectora se puede ver afectada en gran medida.

El comportamiento obtenido se puede justificar teniendo en cuenta la diferente porosidad de los materiales estudiados: a menor porosidad, el recubrimiento es más homogéneo y uniforme $(19,20)$.

En las figuras 3, 4 y 5 se muestran las imágenes obtenidas por microscopía electrónica de barrido (MEB) para los distintos tipos de soporte (granito, mármol y arenisca) originales (a) o recubiertos con HOI (b). En el caso del granito (figura 4) y mármol (figura 5), se observa la superficie totalmente recubierta con una capa continua y uniforme, lo que es debido a que son materiales muy poco porosos y el HOI no penetra en los poros y por lo tanto queda en la superficie del material soporte. Por otro lado, en el caso de la arenisca (figura 6) no se observa dicha uniformidad del recubrimiento puesto que al poseer mayor porosidad el HOI ha penetrado en los poros en mayor o menor medida según sea al tamaño de éstos. Los resultados obtenidos confirman a los obtenidos por ATR anteriormente comentados. 


\subsubsection{Comportamiento antivandálico}

En la figura 7 se presentan las fotografías realizadas con cámara digital convencional a los tres materiales estudiados (granito, mármol y arenisca respectivamente) sin recubrir (a) o recubiertos con HOI (b), después de aplicar la mancha de pintada (c) y, por último, después de la limpieza de la mancha de la pintada (d). En ella se puede ver cómo tanto en granito como en mármol el HOI ha actuado como recubrimiento protector, impidiendo la penetración de la pintada y facilitando su eliminación. Por el contrario, en el caso de la arenisca, tanto el HOI como la pintada son absorbidos por el material, lo que impide la eliminación de la mancha de pintada por el procedimiento empleado. Este resultado de nuevo indica que la porosidad del material pétreo es fundamental a la hora de que el recubrimiento actúe como protector.

\subsubsection{Ensayo de resistencia a la lluvia}

La capacidad antivandálica del recubrimiento estudiado no se ve alterada por el efecto de los ciclos de simulación de

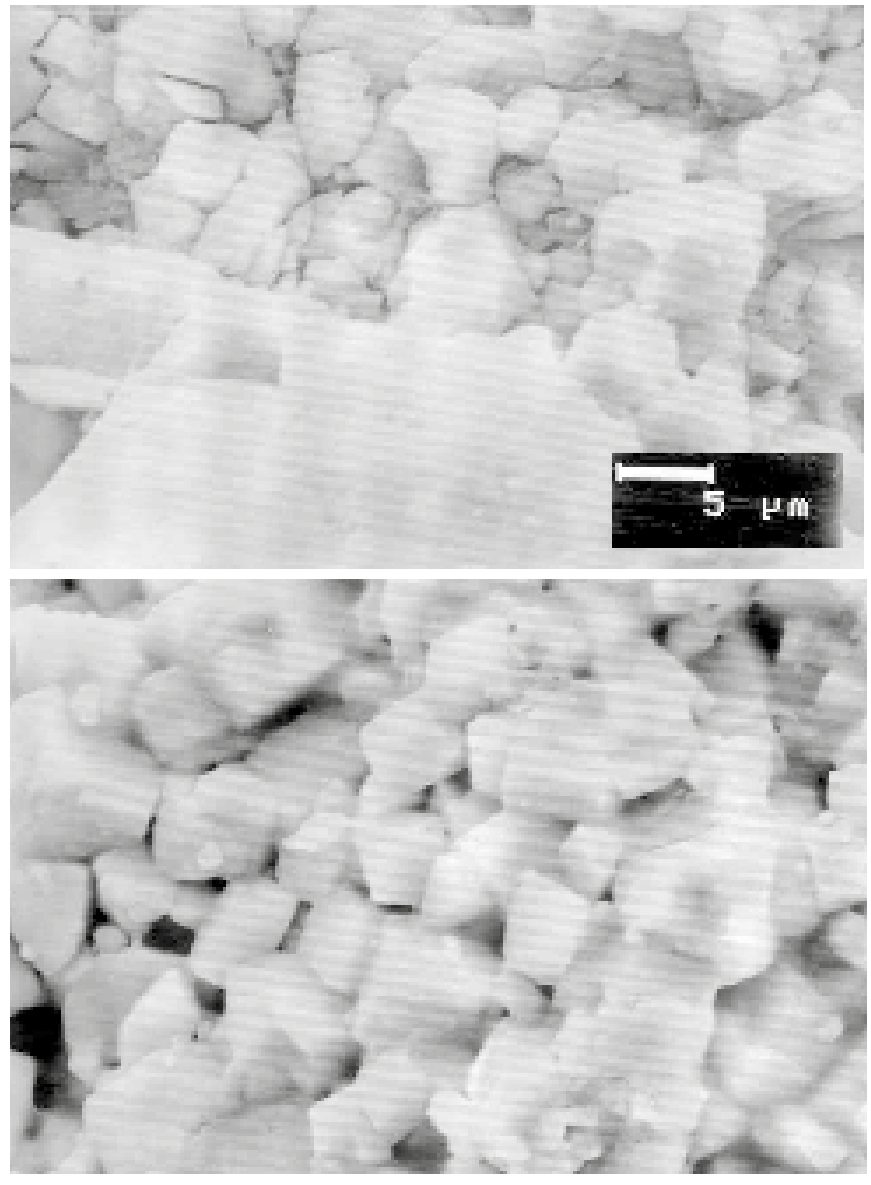

Fig. 6.- Fotografías de microscopía electrónica de barrido de arenisca. (a) original; recubierta con HOI.

lluvia por lo que sólo se muestran, a título de ejemplo, las fotografías correspondientes a los 10 ciclos de lluvia (figura 8). En dicha secuencia se observa cómo la funcionalidad antivandálica persiste tanto en granito como en mármol, ya que la pintura se elimina fácilmente; no así en el caso de la arenisca, donde la pintura queda retenida y no es posible su eliminación. En definitiva, la microestructura y sobre todo la
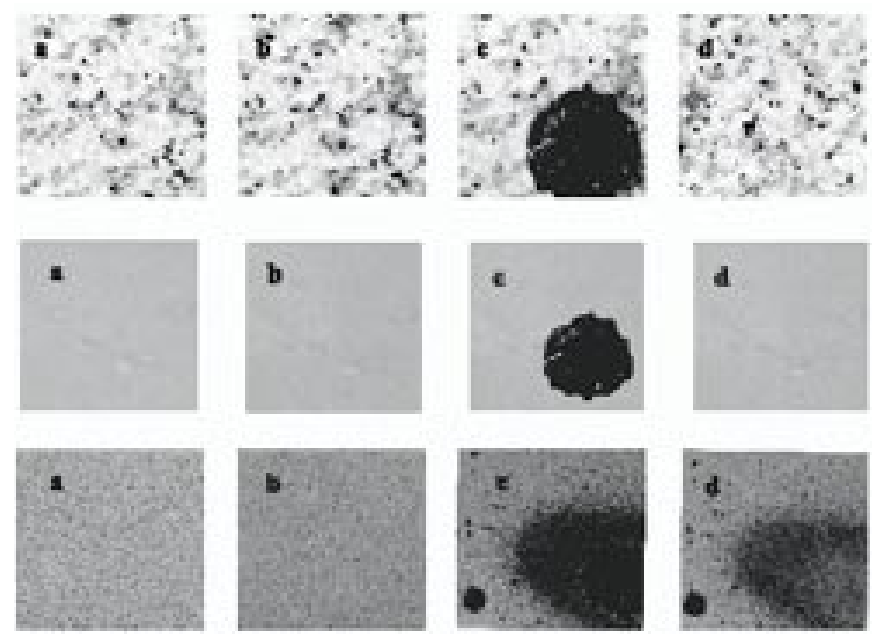

Fig. 7.- Comportamiento antivandálico de las distintas superficies pétreas recubiertas con HOI. Fila 1, Granito; Fila 2, Mármol; Fila 3, Arenisca.

porosidad del material pétreo es determinante a la hora de que el recubrimiento protector posea el efecto deseado (21) .

En la figura 9, se presentan los resultados de los análisis cromáticos obtenidos antes y tras los ciclos de lluvia. En el caso de las dos superficies menos porosas, granito y mármol, la variación cromática del soporte no es apreciable por el ojo humano puesto que los datos experimentales se sitúan en el interior de la elipse de tolerancias. Incluso puede observarse
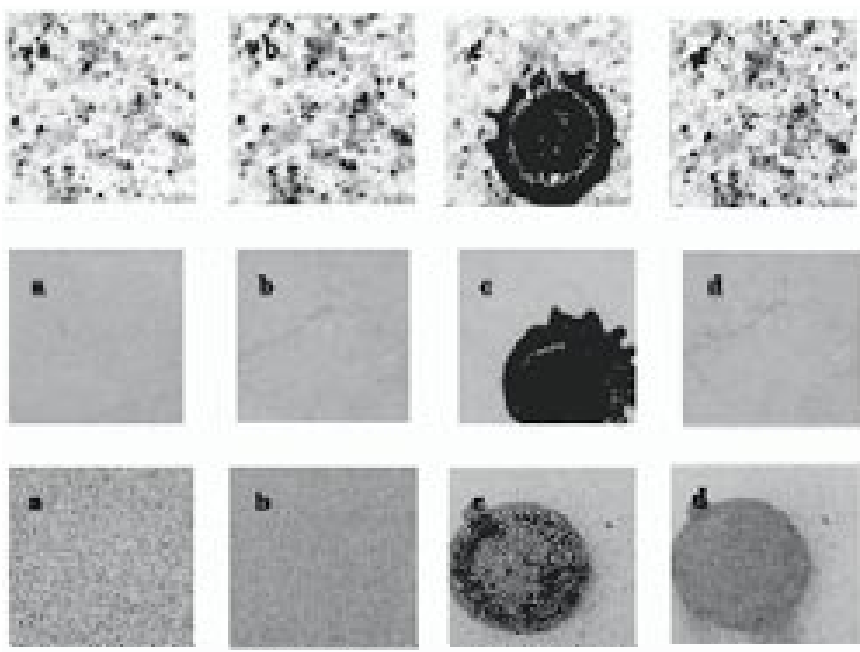

Fig. 8.- Comportamiento antivandálico de las distintas superficies pétreas recubiertas con HOI sometidas a 10 ciclos de lluvia. (a) superficie original recubierta; (b) superficie recubierta sometida a 10 ciclos de lluvia. (c) pintada sobre la superficie recubierta y sometida a 10 ciclos de lluvia. (d) aspecto final-retirada de la pintada.

como en el caso del mármol los puntos experimentales están bastante próximos, excepto para el ensayo de 2 ciclos de lluvia, lo que indica la gran homogeneidad superficial del material en cuanto a color se refiere. Esta homogeneidad es la que ha servido para seleccionar este material como base para los ensayos de envejecimiento por radiación UV tal y como se comentará en el próximo apartado. Por el contrario, para 


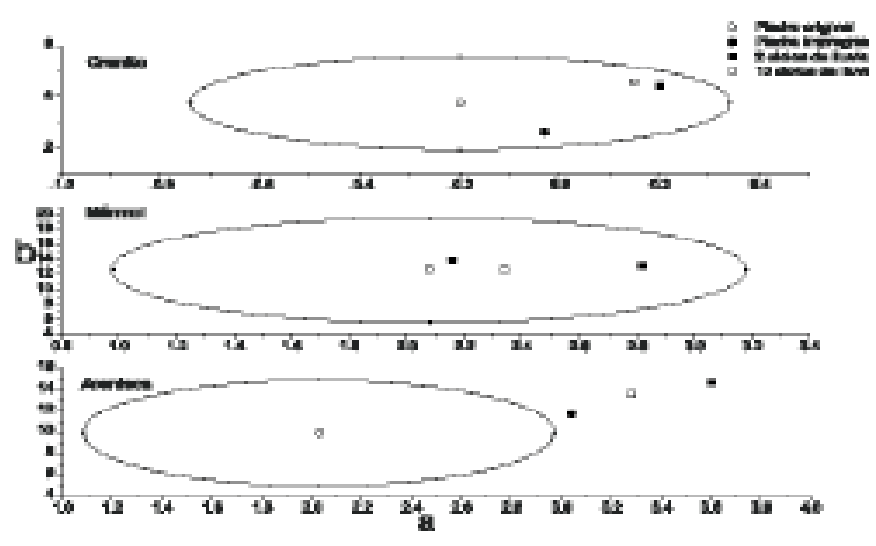

Fig. 9.- Variación cromática del mármol recubierto con HOI; sometido a envejecimiento artificial por exposición a radiación UV.

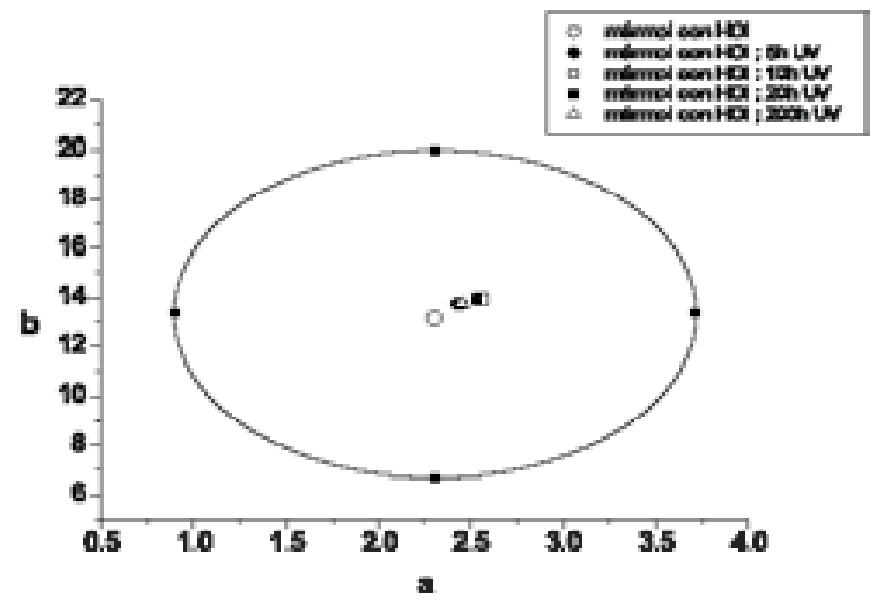

Fig. 10.- Variación cromática de los distintos soportes pétreos recubiertos con HOI y sometidos a 2 y 10 ciclos de lluvia.

la arenisca los datos experimentales se encuentran, en todos los casos, fuera de la elipse de tolerancias, indicando que hay una fuerte variación de color, apreciable a simple vista, incluso antes de ser sometida a los ensayos de resistencia a la lluvia. Esta gran variación de color en el propio material sin impregnar por el recubrimiento antivandálico es debida a la porosidad de la muestra tal y como se había comentado al analizar las curvas de porosimetría de mercurio.

\subsubsection{Ensayo de envejecimiento por luz UV}

Tal y como se ha señalado en el apartado anterior, el mármol fue elegido para analizar el ensayo de envejecimiento del material HOI por radiación UV. Esta elección estuvo en la escasa variación de color que mármol sin recubrir en cualquier parte analizada. Los resultados de los análisis cromáticos del mármol impregnado sometido a los diferentes ciclos de envejecimiento por radiación UV, se muestran en la figura 10. En ella se observa que la variación cromática del recubrimiento es mínima e incluso después de $200 \mathrm{~h}$ de exposición a la radiación UV, el color resultante medido está siempre dentro de la elipse de tolerancias. Por lo tanto el recubrimiento no sufre variación cromática por efecto de la luz UV lo que indicaría su elevada resistencia a la fotodegradación solar.

\section{CONCLUSIONES}

En este trabajo se ha estudiado tanto la capacidad antivandálica de un HOI sobre diferentes soportes pétreos, como la resistencia del tratamiento a la intemperie. Las conclusiones obtenidas son las siguientes:

- Se ha comprobado que la funcionalidad antivandálica del HOI empleado sobre materiales pétreos depende fundamentalmente de las características texturales de los soportes pétreos que determinan el espesor de la capa de protección y la penetración del HOI en dicho material. El material híbrido estudiado presenta un excelente comportamiento antivandálico en aquellos soportes de baja porosidad (granito, mármol), incluso después de someterle a ensayos de envejecimiento, como exposición al agua de lluvia. No sucede así en el caso de materiales de alta porosidad (arenisca), donde la funcionalidad antivandálica es reducida o incluso nula, debido a que el grado de penetración del HOI en el soporte pétreo es tal que no actúa como recubrimiento protector.

- Los estudios cromáticos han demostrado que, en el caso de materiales de baja porosidad como mármol y granito, no hay variación de color de la superficie cuando ésta se recubre con HOI, mientras que en el caso de la arenisca, de elevada porosidad, los cambios de color al impregnar su superficie son detectables incluso para el ojo humano.

- Los estudios cromáticos realizados para evaluar la estabilidad cromática del recubrimiento frente a la exposición a la radiación UV han demostrado que los cambios de color que experimenta dicho recubrimiento están incluidos dentro de la elipse de tolerancias por lo que no son detectables por el ojo humano.

\section{BIBLIOGRAFÍA}

1. A. Moropoulou, N. Kouloumbi, G. Haralamppoulos, A. Konstanti, P. Michailidis. "Criteria and methodology for the evaluation of conservation interventions on treated porous stone susceptible to salt decay". Progress in Organic Coatings 48, 259-270 (2003).

2. G. Zappia, C. Sabbioni, C. Riontino, G. Gobbi, O. Favoni. "Exposure tests of building materials in urban atmosphere". The Science of the Total Environment 224, 235-244 (1998).

3. M. Alvarez de Buergo, R. Fort González. "Protective patinas applied on stony façades of historical buildings in the past". Construction and Building Materials 17, 83-89 (2003).

4. C. Selwitz, E. Doehne. "The evaluation of crystallization modifiers for controlling salt damage to limestone". J. Cultural Heritage 3, 205-216 (2002).

5. B. J. Colston, D. S. Watt, H. L. Munro. "Environmentally-induced stone decay: the cumulative effects of crystallization -hydration cycles on a Lincolnshire oopelsparite limestone". J. Cultural Heritage 4, 297-307 (2001).

6. D. Benavente, M. A. García del Cura, J. García-Guinea, S. Sánchez-Moral, S. Ordóñez. "Role of pore structure in salt crystallisation in unsaturated stone". J. Cryst. Growth 260, 532-544 (2004)

7. D. M. Webley, M. E. Henderson, I. F. Taylor. "Deterioration of natural stone". J. Soil Sci. 14(1) 102-112 (1963)

8. G. C. Borgia, V. Bortolotti, M. Camaiti, F. Cerri, P. Fantazzini, F. Piacenti. "Performance evolution of hydrophobic treatments for stone conservation investigated by MRI". Mag. Res. Imag. 19, 513-516 (2001).

9. 11. M. J. Melo, S. Bracci, M. Camaiti, O. Chiantore, F. Piacenti. "Photodegradation of acrylic resins used in the conservation of stone". Polym. Degrad. Stability 66, 23-30 (1999).

10. 12. M. Álvarez de Buergo Ballester, R. Fort González. “Basic methodology for the assessment and selection of water-repellent treatments applied on carbonatic materials". Progress Organic Coatings. 43, 258-266 (2001).

11. P. Cardiano, P. Mineo, S. Sergi, R.C. Ponterio, M. Triscari, P. Piraino. "Epoxy-silica polymers as restoration materials. Part II". Polymer 44 (2003) 4435-4441 
12. P. Cardiano, R.C. Ponterio, S. Sergi, S. Lo Schiavo, P. Piraino. "Epoxy-silica polymers as stone conservation materials". Polymer 46 (2005) 1857-1864

13. 8. F. Rubio, J. Rubio, J. L. Oteo. "Effect of reaction parameters on the hydrolysis of zirconium propoxide. A study by infrared spectroscopy". J. Mat. Sci. Let. 17, 1839-1842 (1998).

14. 9. S. Murcia-Mascarós, B. Asenjo, I. García-Perulero, J. Rubio, J. L. Oteo. “New Nanocomposites Polyfunctional Materials for Treatment and Protection of Monuments and Buildings". In: The silicates in conservative treatments. Tests, improvements and evaluation of consolidating performance" Ed. IGIIC. Dic. 2002

15. C. Brucherttseifer, S. Bruggerhoff, J. Grobe, K. Stopepek-Langner. “LongTerm Exposure of Treated Natural Stone. Development and First Results of a Testing Concept", F. H. Wittmann, A. J. M. Siemens and L. G. W. Verhoef, eds., Proceedings of the First International Symposium on Surface Treatment of Building Materials with Water Repellent Agents. Delf University of Technology. Faculty of Architecture, Delft, pp. 27/1-27/11. (1995).
16. 14. J. L. Oteo, J. Rubio, F. Rubio; Consejo Superior de Investigaciones Científicas "Materiales termohíbridos inorgánico-orgánicos de baja densidad y su procedimiento de obtención". Patente española núm 9901977 (03-09-1999).

17. 15. M. R. Elvira, L. Alonso, J. Rubio y J. L. Oteo. “Estudio cinético de la reacción de formación de nanocomposites híbridos orgánico-inorgánicos con cationes mixtos ( $\mathrm{Si}-\mathrm{Zr}$ ) a partir de metiltrietoxisilano (MTES)"; presentado en el XLIII Congreso de la Sociedad Española de Cerámica y Vidrio. Pendiente de publicación.

18. Medida y Especificación del Color. Asociación Española para la Calidad.

19. A.C. Iñigo, M.A. Vicente, V. Rives "Weathering and decay of granitic rocks: its relation to their pore network". Mechanics of Materials. 32 (2000) 555-560

20. R. Coppola , A. Lapp, M. Magnani , M. Valli “Non-destructive investigation of microporosity in marbles by means of small angle neutron scattering". Construction and Building Materials 16 (2002) 223-227.

21. P. Lopez-Arce, J. Garcia-Guinea, "Weathering traces in ancient bricks from historic buildings". Building and Environment 40 (2005) 929-941

Recibido: 09.05 .05

Aceptado: 13.05 .05 